\title{
Prevalence of bovine hydatidosis in Kombolcha ELFORA abattoir, North Eastern Ethiopia
}

\author{
Fufa Abunna*, Sisay Fentaye, Bekele Megersa, Alemayehu Regassa \\ School of Veterinary Medicine, Hawassa University, Hawassa, Ethiopia; ${ }^{*}$ Corresponding Author: drfufex@yahoo.com
}

Received 20 April 2012; revised 29 May 2012; accepted 11 June 2012

\section{ABSTRACT}

A study to determine the prevalence of bovine hydatidosis was conducted in Kombolcha ELFORA abattoir. A total of 400 cattle randomly sampled and examined after slaughter for the presence of hydatid cysts in the organs and viscera of the animals using the standard meat inspection procedures. Positive or suspected samples were taken to the parasitology laboratory at the Kombolcha regional laboratory and cyst identification, fertility and viability tests were performed. The statistical analysis showed that there was no difference $(P>0.05)$ between the prevalence of bovine hydatidosis and sex of animals, $P>0.05$. The prevalence of hydatidosis was also found to be significant with the age and origin of the study animals $(P<0.05)$. Of 191 cysts examined, $154(80.63 \%)$ calcified, $1(0.51 \%)$ sterile, and $36(18.85 \%)$ fertile cysts. From the total fertile cysts, $24(72.22 \%)$ were found to be viable. Of 108 cysts recorded in the lung, $74(68.52 \%)$ calcified, $1(0.93 \%)$ sterile, $21(19.44 \%)$ viable and $12(11.11 \%)$ were non viable. Furthermore, of 70 cysts recorded in the liver, 67 $(95.71 \%)$ were found to be calcified and $3(4.29 \%)$ viable. A total of 16,068 cattle were slaughtered from 2003 to August 2007 and the overall 1955 (12.17\%) prevalence of hydatidosis was recorded. The results of this study showed that hydatidosis pose significant economic problems by causing condemnation of considerable numbers organs, rendering them unfit for market. Therefore, initiation and implementation of control measures is necessary in order to alleviate its economic impact as well as zoonotic risks to the human.

Keywords: Abattoir; Hydatidosis; Cattle; Prevalence; Kombolcha; Ethiopia

\section{INTRODUCTION}

Echinococcosis is a zoonotic infection caused by Echinococcus granulosus, which is a small tapeworm and in the natural cycle, dogs and other canids are typical definitive hosts and ungulates, including sheep, goats, pigs and horses, are intermediate hosts in which hydatid cysts occur. The latter stage can also develop in humans [1]. Although E. granulosus penetrates deep between the villi of the small intestine of the definitive host, there are no pathogenic effects even in heavy infections, suggesting that infected definitive hosts are asymptomatic carriers of the parasite. Furthermore, infections with $E$. granulosus cysts in the intermediate host are typically asymptomatic, except for a small number of cases with chronic and heavy infections. There are no reliable methods for the routine diagnosis of infections in living animals, but in rare cases cysts have been identified by ultrasonography alone or in conjunction with serum antibody detection [2]. A new ELISA with a high specificity and a sensitivity of $50 \%$ to $60 \%$ might be useful for detecting E. granulosus cysts in sheep on a flock basis but cannot be used for a reliable diagnosis of infected individuals [3]. The most reliable diagnostic method is cyst detection during meat inspection or at post-mortem examination. Cystic echinococcosis in farm animals causes considerable economic problems due to loss of edible livers. Significant losses of meat and milk production and fleece values from infected sheep may also occur. These losses are of special significance in countries with low economic outputs where sheep production is of particular importance [4].

A number of studies have conclude that cystic hydatid disease caused by E. granulosus can result in a $10 \%$ decrease in whole of life performance for infected animals (reduction in quality of meat, production of fibre, production of milk and in number of surviving offspring) [5]. However, these figures are not well-known to third world farmers, and are probably insignificant in the face of pandemics of infectious disease and losses due to reproductive diseases, parasitism and starvation. Therefore, all programs of hydatid control around the world have been 
driven by the zoonotic status of the parasite and concerns for human health [6,7].

The scanning surveys were well accepted by the people and provided evidence for the need to expand the present hydatid control program in Turkana to cover the whole hyper endemic focus. Such a program must contain an educational component for, although most groups recognized hydatid cysts, there was complete lack of knowledge concerning the parasite and its mode of transmission [8]. Survey of echinococcosis in eastern Ethiopia by [9], indicated that a total of 171 adult cattle and nine stray dogs were examined for E. granulosus in eastern Ethiopia. Prevalence of $20.5 \%$ and $22 \%$ were recorded in cattle and dogs, respectively.

Currently, some modern export abattoirs are established and have started exporting meat to Middle East countries and this export market is highly promising and is enabling the earning of foreign currency, which is highly needed by the country. Such export market may be influenced by the quality of meat produced especially from diseases. Some of Metacestods which occur in the carcass and organs of ruminants can affect the meat export market either directly or indirectly by causing rejection of carcass/organs for aesthetic reasons by the importer countries or due to condemnation of organs such as liver and heart which are highly demanded by importers. Few reports are available on the prevalence of hydatidosis in North eastern parts of Ethiopia. Therefore, this study was undertaken to determine the prevalence of hydatidosis in cattle slaughtered in Kombolcha ELFORA abattoir.

\section{Materials and Methods}

\subsection{Study Area}

The study was conducted at ELFORA Kombolcha industrial abattoir, located at about $375 \mathrm{~km}$ north east of Addis Ababa at an altitude of 1500 - 1840 m.a.s.l. The area receives an average rainfall and temperature of 750 $\mathrm{mm}-900 \mathrm{~mm}$ and $25^{\circ} \mathrm{C}-30^{\circ} \mathrm{C}$, respectively. Kombolcha is one of the 21 districts of South Wollo administrative zone which shares border with Dessie zuria and Tewuledere districts in the North, with Dessie Zuria and Dessie town in the West, with Kalu and Dessie zuria in the South and Kalu in the East. The district is divided into five urban Kebeles and six rural Kebeles. As estimated by the District Office of Agriculture (DOA), the district has a total area of $78.6 \mathrm{~km}^{2}$. About $33.6 \%$ of the district's area is under crop production, and $1.47 \%$ is serving as a grazing land.

\subsection{Study Population}

Cattle that have been brought to the abattoir for routine meat inspection during the past five-year period was used. Cattle that were presented to the abattoir during routine meat inspection procedure were included.

\subsection{Sample Size Determination}

The number of bovine carcasses examined during slaughtering at the Abattoir during the past five years was used for retrospective abattoir survey. In active abattoir survey, a cross-sectional study type of investigation was conducted and the sample size was determined according to [10] by taking $50 \%$ prevalence. Thus, 385 bovine were sampled. But to increase the sample size, 400 animals were examined.

\subsection{Study Methodology}

Retrospective abattoir data collection: A 5-year data was analyzed for the prevalence of bovine hydatidosis.

Examination of slaughter animals (active abattoir survey): The abattoir survey was conducted in routine examination of arbitrarily selected cattle at the study area. A thorough post-mortem examination was conducted on animals slaughtered at municipal abattoir. The lungs, liver, heart, spleen and kidneys were examined. Echinococcus cysts identified were removed and collected in polyethylene bags or Petri-dish. Each polyethylene bag or Petri-dish was used for hydatid cysts obtained from one animal and was labeled appropriately to show the species. The cysts were transported to the nearest laboratory and examined within one hour.

Cyst fertility and viability: Individual cysts were grossly examined for any evidence of degeneration and calcification. Cysts were selected for fertility studies and to reduce intra-cystic pressure, the cyst wall was penetrated with a needle and opened up with a scalpel and scissors. The contents were then transferred into a sterile container and examined microscopically $(40 \times)$ for the presence of protoscoleces. Cysts which were not containing any protoscoleces as well as heavily supurative or calcified were considered unfertile. The viability of protoscoleces was assessed by the motility of flame cells together with staining with a $0.1 \%$ aqueous eosin solution. Living protoscoleces did not take up the stain, unlike the dead ones [8].

\subsection{Data Management and Analysis}

Abattoir data was collected and recorded on specially designed forms and preliminary analysis was done in Microsoft Excel (Microsoft Corporation). The outcome variables for the abattoir study were cases of "Echinococcosis" detected during routine postmortem inspection at the abattoir. The STATA software program was applied for the statistical analysis of the data obtained from the study. The prevalence of hydatidosis was calculated as 
the number of positive observation divided by the total sample size multiplied by 100 . Logistic regression was used to expressed results and compare risk factors (to determine infection rate on sex, age, origin and body condition basis).

\section{Results}

Active abattoir survey: Of the 400 bovine animals examined during routine meat inspection at ELFORA Kombolcha industrial Abattoir, 68(17\%) were found to be infected with hydatidosis.

Sex: Thirty eight $(9.5 \%)$ from a total 245 female animals and 30(7.5\%) from 155 male animals were positive for bovine hydatidosis. The statistical analysis showed that there was no significant difference $(P>0.05)$ between the prevalence of bovine hydatidosis and sex of animals presented to the abattoir during the study period. $\mathrm{OR}=1.27, P>0.05,95 \% \mathrm{CI}=0.67-2.03$ (Table 1).

Age: Statistical analysis showed that the prevalence of hydatidosis was significant with the age of the study animals, $P<0.05$ and $\mathrm{OR}=1.883418$ and $95 \%=1.08-$ 3.29 (Table 2).

Origin of animals: The prevalence of hydatidosis was statistically significant with the origin of the study animals, higher in animals of highland origin $P<0.05$, OR $=3.418357$ and $95 \%=1.94-6.02$ (Table 3).

Prevalence of hydatidosis and body condition of the animals: The majority of animals presented to the abattoir were poor in body condition and of 400 animals, 1 and $16 \%$ prevalence of hydatidosis were recorded in good and poor body condition animals, respectively (Table 4 ).

The proportion of organs presented on Table 5 shows that the majority of cysts were recorded in the lungs of the study animals.

Fertility and viability study: A total of 191 cysts were collected at the abattoir during the study period. Of the total cysts collected, 24(12.6\%) were found to be alive and 167(87.43\%) were found to be dead (Table 6). A total of 191 cysts were examined in the abattoir

Table 1. Sex distribution of animals infected with hydatidosis.

\begin{tabular}{cccc}
\hline Sex & Total no. of animals examined & Total no. of animals positive for hydatidosis & Prevalence \\
\hline Male & 155 & 30 & $19.35 \%$ \\
Female & 245 & 38 & $15.51 \%$ \\
Total & $\mathbf{4 0 0}$ & $\mathbf{6 8}$ & $\mathbf{1 7 \%}$ \\
\hline
\end{tabular}

Table 2. Prevalence of hydatidosis in cattle in different age groups.

\begin{tabular}{cccc}
\hline Age (years) & Total no. of animals examined & Total no. of animals positive for hydatidosis & Prevalence (\%) \\
\hline$\leq 5$ & 45 & 3 & 6.66 \\
$5-10$ & 300 & 52 & 17.33 \\
$>10$ & 55 & 13 & 23.64 \\
Total & $\mathbf{4 0 0}$ & $\mathbf{6 8}$ & $\mathbf{1 7 \%}$ \\
\hline
\end{tabular}

Table 3. Geographical distribution of hydatidosis in cattle.

\begin{tabular}{cccc}
\hline Origin of the animals & Total no. of animals examined & Total no. of animals positive for hydatidosis & Prevalence (\%) \\
\hline Low land & 221 & 22 & 9.54 \\
High land & 179 & 46 & 25.70 \\
Total & $\mathbf{4 0 0}$ & $\mathbf{6 8}$ & $\mathbf{1 7}$ \\
\hline
\end{tabular}

Table 4. Prevalence of hydatidosis in cattle in relation with their body condition.

\begin{tabular}{cccc}
\hline Body condition of the animals & Total no. of animals examined & Total no. of animals positive for hydatidosis & Prevalence (\%) \\
\hline Good & 36 & 4 & 11.11 \\
Poor & 364 & 64 & 17.58 \\
Total & $\mathbf{4 0 0}$ & $\mathbf{6 8}$ & $\mathbf{1 7}$ \\
\hline
\end{tabular}


Table 5. Proportion of organs infected with bovine hydatidosis.

\begin{tabular}{ccccc}
\hline Lung & Liver & Kidney & Heart & Spleen \\
\hline $50(12.5)$ & $17(4.25 \%)$ & $4(1 \%)$ & $1(0.25 \%)$ & $1(0.25 \%)$ \\
\hline
\end{tabular}

Table 6. Proportion of viable cysts in different organs inspected.

\begin{tabular}{ccc}
\hline Organs inspected & Total cysts recorded & Viable cysts \\
\hline Lung & 108 & $21(19.4 \%)$ \\
Liver & 70 & $3(4.3 \%)$ \\
Heart & 1 & $0(0 \%)$ \\
Kidney & 11 & $0(0 \%)$ \\
Spleen & 1 & $0(0 \%)$ \\
Total & $\mathbf{1 9 1}$ & $\mathbf{2 4 ( 1 2 . 6 \% )}$ \\
\hline
\end{tabular}

during the study period. Of the total cysts examined, $154(80.63 \%)$ calcified, $1(0.51 \%)$ sterile, and $36(18.85 \%)$ fertile. From the total fertile cysts identified, 24(72.22\%) were found to be viable cysts (Table 7). The cyst condition by the organ of involvement was found to be: in lung from the total 108 cyst recorded, 74(68.52\%) calcified $1(0.93 \%)$ sterile, $21(19.44 \%)$ viable and $12(11.11 \%)$ were non viable fertile cysts. In the liver, out of 70 cysts, 67(95.71\%) were calcified and 3(4.29\%) were viable. The remaining organs, kidney 11 cysts were calcified only and also in the heart and spleen a single cyst was found with calcification. From the inspected organs at the abattoir, 19 animals were found to harbor more than 5 to 20 cysts and one animal harbored 21 hydatid cysts. Large cyst sizes were recorded in the lungs of animals observed in the abattoir (Table 8).

Logistic regression of the selected risk factors for the hydatidosis infection: The logistic regression (Table 9) of the selected risk factors for bovine hydatidosis was assessed, thus age and origin of the animals were found to statistically significant.

Retrospective abattoir survey: Meat inspection records in an abattoir located in ELFORA Kombolcha during a 5-year period from September, 2003 to August 2007 were used to determine the total number of organs condemned due to hydatidosis in cattle. A total of 16,068 cattle slaughtered in the 5-year period and the overall 1955(12.17\%) and 952(5.92\%) of lung and liver were condemned due to hydatidosis, respectively (Table 10).

\section{Discussion}

E. granulosus shows considerable geographic variations. Apart from aboiotic variables, factors such as difference
Table 7. Distribution of cyst fertility and viability in different organs in cattle.

\begin{tabular}{cccccc}
\hline Organ & \multicolumn{5}{c}{ Cyst condition } \\
\hline & \multicolumn{2}{c}{ Fertile } & \multicolumn{2}{c}{ Non fertile } & \\
\cline { 2 - 4 } & Viable & Non viable & Sterile & Calcified & Total \\
\hline Lung & 21 & 12 & 1 & 74 & 108 \\
Liver & 3 & 0 & 0 & 67 & 70 \\
Kidney & 0 & 0 & 0 & 11 & 11 \\
Heart & 0 & 0 & 0 & 1 & 1 \\
Spleen & 0 & 0 & 0 & 1 & 1 \\
Total & $\mathbf{2 4}$ & $\mathbf{1 2}$ & $\mathbf{1}$ & $\mathbf{1 5 4}$ & $\mathbf{1 9 1}$ \\
\hline
\end{tabular}

Table 8. Distribution of cysts in different organs based on their size in cattle.

\begin{tabular}{ccccccccccc}
\hline \multicolumn{10}{c}{ Cyst size $^{*}$} \\
\hline Organs & Small & Medium & Large & Calcified cysts & \multicolumn{1}{c}{ Total } \\
\hline & No. & $\%$ & No. & $\%$ & No. & $\%$ & No. & $\%$ & No. & $\%$ \\
Lung & 22 & 100 & 8 & 72.7 & 4 & 100 & 74 & 48.1 & 108 & 56.5 \\
Liver & 0 & 0 & 3 & 27.3 & 0 & 0 & 67 & 43.5 & 70 & 36.7 \\
Kidney & 0 & 0 & 0 & 0 & 0 & 0 & 11 & 7.1 & 11 & 5.8 \\
Heart & 0 & 0 & 0 & 0 & 0 & 0 & 1 & 0.65 & 1 & 0.5 \\
Spleen & 0 & 0 & 0 & 0 & 0 & 0 & 1 & 0.65 & 1 & 0.5 \\
Total & 22 & 100 & 11 & 100 & 4 & 100 & 154 & 100 & 191 & 100 \\
${ }^{*}<4$ cm $=$ small, $4-6 \mathrm{~cm}=$ medium and $>6 \mathrm{~cm}=1 \operatorname{large}$. & &
\end{tabular}

Table 9. The logistic regression analysis of the risk factors for bovine hydatidosis at ELFORA Kombolcha abattoir in 20072008.

\begin{tabular}{llllll}
\hline $\begin{array}{l}\text { Risk } \\
\text { factors }\end{array}$ & $\begin{array}{l}\text { Odds } \\
\text { ratio }\end{array}$ & Std. Err. & $\mathbf{Z}$ & $\boldsymbol{P}>|\mathbf{z}|$ & $\begin{array}{l}\text { 95\% Conf. } \\
\text { Interval }\end{array}$ \\
\hline Sex & 1.164716 & 0.3299127 & 0.54 & 0.590 & $0.67-2.03$ \\
Age & 1.883418 & 0.5380605 & 2.22 & 0.027 & $1.08-3.29$ \\
Origin & 3.418357 & 0.9865505 & 4.26 & 0.000 & $1.94-6.02$ \\
$\begin{array}{l}\text { Body } \\
\text { condition }\end{array}$ & 0.4768376 & 0.2717546 & -1.30 & 0.194 & $0.16-1.465$ \\
\hline
\end{tabular}

Table 10. The number of liver and lung condemned due to hydatidosis in cattle slaughtered at ELFORA Kombolcha abattoir from 2003-2007.

\begin{tabular}{lcccccc}
\hline Year & $\mathbf{2 0 0 3}$ & $\mathbf{2 0 0 4}$ & $\mathbf{2 0 0 5}$ & $\mathbf{2 0 0 6}$ & $\mathbf{2 0 0 7}$ & Total \\
\hline $\begin{array}{l}\text { Total no. of } \\
\text { animals slaughtered }\end{array}$ & 2741 & 259 & 4580 & 2238 & 6250 & 16,068 \\
$\begin{array}{l}\text { Total no. of } \\
\text { liver condemned }\end{array}$ & 221 & 18 & 323 & 90 & 300 & 952 \\
$\begin{array}{l}\text { Total no. of } \\
\text { lungs condemned }\end{array}$ & 471 & 42 & 608 & 202 & 632 & 1955 \\
\hline
\end{tabular}


in culture social activities religious facts and attitude to dogs in different regions contribute to the variations in the prevalence rate. In the present study, the prevalence of hydatid disease in the study area was $17 \%$, which is relatively lower than reports by $[9,11,12], 20.5 \%, 28.3 \%$, $46.5 \%, 25.7 \%$ in cattle slaughtered in Eastern Ethiopia, Kombolcha, Debre Zeit, South Omo, respectively.

The finding record in this study is important and these are governed by the following events: most cattle are slaughtered at their older age when they become exhausted in milk and/or calf production or when they have reduce capacity to work through which they have a greater chance to be infected with E. granulosus. In the rural area most farmers keep at least one dog for guarding the house and the livestock. This is one way the accesses to gain infected lungs and other organs to dogs. Meat inspection is practiced only at few sites and there is no proper disposal of condemned organs except at Sopral Kombolcha meat factory, where condemned organs and carcasses are further processed for animal feed and it is not source of infection for E. granulosus. There is lack of awareness by the people of the health hazard as well as the economic importance the disease. The statistical analysis showed that the prevalence of hydatidosis was significant with the age of the study animals (Tables 2 and $\mathbf{9}), P<0.05$, especially for the middle age groups are affected more. This may be due to the fact that cattle are slaughtered at their medium or older age with which they have greater chance of being infected with E. granulosus. And the growth of the hydatid is slow, maturity being reached in 6 - 12 months [13].

The prevalence of hydatidosis was statistically significant with the origin of the study animals, higher in animals of high land than the lowlands $P<0.05$. The eggs tolerate a wide temperature range: heat damage does not appear to occur until temperature reach $38^{\circ} \mathrm{C}$ and the eggs withstand a temperature as low as $-30^{\circ} \mathrm{C}$ [6]. Within certain limits the longevity of the eggs decreases as the temperature increases according to [11]. This may be due to the fact that, the religious factor, in the most lowland areas of Ethiopia, the Muslim people are more privileged and dog considered as "NEJASSA" on there religious and "Qurran" basis. So that dog is rarely domesticated and reduces the infection rate of cattle in the lowland area.

The statistical analysis should that there was no significant difference between the prevalence of bovine hydatidosis and sex of the study animals. All exposed groups are get access to infection with different degree based on the organ parasitized and the size attained by hydatid cyst. The incidence of the disease (infection) in the area both in animals and man can be determined from its level in dogs. It is also valuable to investigate the management, and feeding of dogs to discover how the cycle of infection maintained [11]. Habit, customs and occupation, particularly attitude to dogs, may also influence the prevalence of metacestodes infection in a certain group [12].

The prevalence of hydatidosis was statistically not significant with the body condition of the animal. Kombolcha ELFORA abattoir is an industrial abattoir, poor body condition cattle are preferable than fattened animals to prolong the shelf life of the canned meat. The majority of the study animals presented to the abattoir were poor body condition and of 400 animals $4(1 \%)$ and $64(16 \%)$ prevalence of hydatidosis were recorded in good and poor body condition animals respectively (Table 4).

The liver and lung are the most commonly infected organs. The kidney, heart and spleen are the least affected organs in the study animal. Similar findings were also obtained by various workers and it's indicated that the liver and lungs are the most commonly affected organs with hydatid cyst due to the reason that there are the first large capillary fields encountered by the blood borne onchospheres. However, development of $H$. cysts occurs occasionally in other organs and tissue when onchosphere escape in to the general systemic circulation [13]. From the organ prevalence study the lung is found to be the most commonly affected organ followed by liver. The liver infection may be a reflection of the route of parasite entry and seems to support the hypotheses of heapatic portal distribution of the onchospheres leading to the liver infection. The proportion of large medium and small size cysts is higher in the lung than in the liver. Similar finding was obtained by [11] in the same study area. Similarly in the rare sites such as the abdominal cavity, where unrestricted growth is possible, the hydatid may attain very large size containing several liters of fluid [13]. The percentage of calcified cysts is found to be higher in the liver than in the lungs. This may be associated with the relatively higher reticulo-endothelial cells and abundant connective tissue reaction of the organ [6], which encapsulates the cyst within a fibrous wall up to $13 \mathrm{~mm}$ thick. From the fertility and viability study, the percentage of fertile cysts was found higher in lung than the liver, this is due to the relatively softer consistency of the lung allows easer development of the pressure cyst and fertility of hydatid cyst may show tendency to increase. Meat inspection records in an abattoir during a 5-year period from September, 2003 to August 2007 indicated that from the total of 16,068 cattle slaughtered, 1955(12.17\%), 952(5.92\%) lung and liver were condemned due to hydatidosis. The number of condemned organs is smaller when compared to other researchers. This might be true that in the abattoir they record only the totally condemned organs.

Hydatidosis causes considerable public health hazard. It is well known that an efficient meat inspection service 
should function as an important monitoring agent in the control of animal diseases with considerable economic and public health significance mainly in cases of chronic and ill-defined conditions which are not apparent either to the livestock owners or Veterinary surgeon. More importantly a feedback from the slaughterhouses to the individual farm is of great value in the field of preventive medicine. Therefore, the establishment of well equipped standardized abattoirs, creation of public awareness in terms of knowledge of zoonotic importance of hydatidosis and control of stray dogs in order to minimize the risk of acquiring the most important zoonotic diseases are paramount importance.

\section{ACKNOWLEDGEMENTS}

The research was conducted with the willingness and cooperation of abattoir workers and owners of the slaughtered animals. All contributions are gratefully acknowledged.

\section{REFERENCES}

[1] Eckert, J., Deplazes, P., Craig, P.S., Gemmell, M.A., Gottstein, B., Heath, D., Jenkins, D.J., Kamiya, M. and Lightowlers, M. (2001) Echinococcosis in animals: Clinical aspects, diagnosis and treatment. In: Eckert, J., Gemmell, M.A., Meslin, F.X. and Pawlowski, Z.S., Eds., WHO/ OIE Manual on Echino-Coccosis in Humans and Animals: A Public Health Problem of Global Concern, World Organization for Animal Health, Paris, 72-99.

[2] Eckert, J. and Deplazes, P. (2004) Biological, epidemiological, and clinical aspects of Echinococcosis, a zoonosis of increasing concern. Clinical Microbiology Reviews, 17, 107-135. doi:10.1128/CMR.17.1.107-135.2004

[3] Kittelberger, R., Reichel, M.P., Jenner, J., Heath, D., Lightowlers, M.W., Moro, P., Ibrahem, M.M., Craig, P.S. and O'Keefe, J.S. (2002) Evaluation of three enzymelinked immunosorbent assays (ELISAs) for the detection of serum antibodies in sheep infected with Echinococcus granulosus. Veterinary Parasitology, 110, 57-76. doi:10.1016/S0304-4017(02)00308-4

[4] Torgerson, P.R., Dowling, P.M. and Abo-Shehada M.N. (2001) Estimating the economic effects of cystic echino- coccosis. Jordan, a developing country with lower-middle income. Annals of Tropical Medicine and Hygiene, 95, 595-603.

[5] Battelli, G. (1997) Evaluation of the economic costs of Echinococcosis. Arch Inter Hyd, 32, 33-37.

[6] Gemmell, M.A., Roberts, M.G., Beard, T.C., Campano, Diaz, S., Lawson, J.R. and Nonnemaker J.M. (2001) Control of Echinococcus granulosus. In: Eckert, J., Gemmell, M.A., Meslin, F.-X. and Pawlowski, Z.S., Eds., WHO/OIE Manual on Echinococcosis in Humans and Animals: A Public Health Problem of Global Concern, World Organisation for Animal Health, Paris, 195-203.

[7] Economedes, P., Larrieu, E.J. and Orlando, D. (2001) Evolution of programmes for control of Echinococcus granulosus (examples). In: Eckert, J., Gemmell, M.A., Meslin, F.X. and Pawlowski, Z.S., Eds., WHO/OIE Manual on Echinococcosis in Humans and Animals: A Public Health Problem of Global Concern, World Organisation for Animal Health, Paris, 204-209.

[8] Macpherson, C.N., Spoerry, A., Zeyhle, E., Romig, T. and Gorfe, M. (1989) Pastoralists and hydatid disease: An ultrasound scanning prevalence survey in east Africa. Transactions of the Royal Society of Tropical Medicine and Hygiene, 83, 243-247. doi:10.1016/0035-9203(89)90664-0

[9] Mersie, A. (1993) Survey of echinococcosis in eastern Ethiopia. Veterinary Parasitology, 47, 161-163. doi:10.1016/0304-4017(93)90187-R

[10] Thrusfield, M. (1995) Sampling. In: Veterinary Epidemiology, 2nd Edition, Black Well Science Ltd., London, 179284.

[11] Asrat, G. (1996) Prevalence and economic significance of hydatidosis/echinococcosis in slaughtered cattle, sheep and got in South Wollo. DVM thesis, Addis Ababa University, Debre Zeit, Ethiopia.

[12] Jobre, Y., Lobago, F., Tiruneh, R., Abebe, G. and Dorchie, P. (1996) Hydatidosis in three selected region in Ethiopia: An assessment trial on its prevalence, economic and public health importance. Revue de Medicine Veterinaire, 147, 797-804.

[13] Urquhart, G.M., Duncan, J., Armour, L., Dunn, J. and Jenning, A.M. (1996) Veterinary parasitology. 2nd Edition, Blackwell Science, Oxford, 120-129. 